\title{
Interactive Antimicrobial Profiles of Astragalus membranaceus (Fisch.) Bunge Extracts and Conventional Antibiotics against Pathogenic and Non-pathogenic Gastrointestinal Bacteria
}

\author{
Wenjing Lai ${ }^{1,2}$, Ian Edwin Cock ${ }^{3,4}$, Matthew James Cheesman ${ }^{1,5^{*}}$ \\ 'School of Pharmacy and Pharmacology, Gold Coast Campus, Griffith University, Parklands Drive, Southport, Queensland 4222, AUSTRALIA \\ ${ }^{2}$ College of Traditional Chinese Medicine, Guangdong Pharmaceutical University, Guangzhou 510006, CHINA \\ ${ }^{3}$ School of Environment and Science, Nathan Campus, Griffith University, 170 Kessels Rd, Nathan, Queensland 4111, AUSTRALIA \\ "Environmental Futures Research Institute, Nathan Campus, Griffith University, 170 Kessels Rd, Nathan, Queensland 4111, AUSTRALIA. \\ ${ }^{5}$ Menzies Health Institute Queensland, Quality Use of Medicines Network, Queensland 4222, AUSTRALIA.
}

\begin{abstract}
Background: The aim of this project was to investigate the efficacy of Astragalus membranaceus (Astragalus) extracts, alone and in combination with conventional antibiotics, against diarrhoea- and dysentery-causing pathogens, as well as against non-pathogenic gastrointestinal bacterial strains. The study sought to validate the chemotherapeutic potential of a traditional Chinese medicinal plant and to identify combinational therapies with increased efficacy compared to either the extracts or conventional antibiotics alone. Methods: Astragalus root powder was extracted with solvents of varying polarity and screened for inhibition of bacterial growth. Susceptibility was assessed by disc diffusion techniques, whilst the minimum inhibitory concentrations (MICs) were quantified by liquid dilution assays. To screen for combinatorial effects, the Astragalus root extracts were combined with a range of conventional antibiotics and tested against each bacterial strain using liquid dilution assays. $\Sigma \mathrm{FIC}$ values were determined and used to determine the class of interaction. Results: Aqueous Astragalus root extracts did not significantly inhibit the growth of the non-pathogenic or beneficial gut microflora bacteria E. cloacae, E. coli or $E$. faecalis, but possessed mild inhibitory activity against pathogenic $A$. faecalis, A. hydrophila, B. cereus, S. newport and S. sonnei bacteria. Combinations of the Astragalus extracts and conventional antibiotics generally
\end{abstract}

\section{INTRODUCTION}

The World Health Organization (WHO) has reported that more than half a million children under the age of 5 die each year from diarrhoeal disease, ${ }^{1}$ equating to over 1400 deaths per day. Diarrhoea remains a leading killer of children, accounting for $8 \%$ of all death among children of this age group. ${ }^{2}$ Exacerbating the problem are bacteria that have developed resistance to conventional antibiotics, rendering these medicines ineffective against diarrhoea-causing pathogens..$^{1,3-5}$ As such, an urgent need has arisen for the development of new treatment options to combat these illnesses, either through novel drug molecules or by utilizing new therapy regimens that enable, or re-purpose, previously effective antibiotics that have lost their potency due to the emergence of highly resistant infections. Many plants used in traditional medicinal systems are effective in the treatment of diarrhoea. Traditional Chinese Medicine (TCM) has been used for thousands of years as a method for treating a variety of different ailments and is a prominent feature of Chinese history and culture. ${ }^{6}$ Traditional healing practices have become increasingly accepted by people in many regions of the world in recent years, with more researchers now exploring the scientific merit of TCM. Astragalus membranaceus (Fisch.) Bunge or Astragalus; (also known as Huangqi - 黄芪/黃芪 is one of the most widely used Chinese medicinal plants ${ }^{7}$ (Figure 1a) and is mainly grown in the northern regions of China as well as in Mongolia and Siberia. ${ }^{8}$ In recent years, the chemical composition and the pharmacological activity of Astragalus have been studied extensively produced additive or indifferent interactions, indicating that they are safe to use concomitantly without compromising the efficacy of either component. Two cases of antagonistic combinations were detected against $B$. cereus and S. sonnei. Conclusion: Mild inhibition of 5 pathogenic bacteria occurred with aqueous Astragalus extracts, with a number of additive and antagonistic interactions arising when tested in combination with conventional antibiotics. Astragalus may be used safely in the presence of normal gut bacteria and in most combinations with conventional antibiotics. Key words: Traditional Chinese Medicine (TCM), Fabaceae, Astragalus, Disc diffusion, Liquid dilution assays, Combinational therapies, Diarrhea, Synergy.

\section{Correspondence:}

Dr. Matthew J. Cheesman ${ }^{1,2}$

'School of Pharmacy and Pharmacology, Gold Coast Campus, Griffith University,

Parklands Drive, Southport, Queensland 4222, AUSTRALIA.

${ }^{2}$ Menzies Health Institute Queensland, Quality Use of Medicines Network,

Queensland 4222, AUSTRALIA.

Phone no: +61755529230

E-mail: m.cheesman@griffith.edu.au

DOI: $10.5530 / p c .2018 .4 .33$
(Figure 1b-e) ${ }^{9-10}$ Chemical analysis of the plant revealed that Astragalus is predominantly composed of polysaccharides, triterpenes and flavonoids. ${ }^{11}$ Pharmacological evidence suggests that Astragalus possesses therapeutic benefits for the immune system and for liver and kidney protection, whilst also having anti-tumorigenic, antiviral, anti-hypertensive and antidiabetic properties. ${ }^{7,12}$ Astragalus has also been incorporated into conventional medicines to treat chronic kidney disease, diabetes, breast cancer, neurodegenerative and coronary diseases. ${ }^{13-15}$

The mechanism of action of Astragalus plant extracts has recently been the focus of considerable investigation. For example, the inhibitory effects of the plant on peritoneal fibrosis may involve both MCP-1 and TGF- $\beta /$ Smad pathways. $^{16}$ Additionally, extracts from Astragalus inhibit breast cancer cells proliferation via PI3K/AKT/mTOR signaling pathway. ${ }^{17-18}$ Astragalus can also regulate iNOS activity of macrophages in different states in vitro by Astragalus polysaccharides. ${ }^{19-20}$ However, studies on the antibacterial properties of Astragalus are lacking. Since the herb is predominantly ingested, thereby reaching sections of the gastrointestinal (GI) tract where both normal gut flora and disease-causing bacteria co-exist, there is a need to investigate the effects of Astragalus on gastrointestinal bacterial strains. Inhibition of the growth of these strains may be beneficial in treating pathogenic disease. However, an understanding of the antibacterial effects of Astragalus plant extracts on normal gut flora is also important as Astragalus extracts are ingested by members of the community for a wide spectrum of ailments unrelated to bacterial infection. Bacterial strains which are non-pathogenic and 


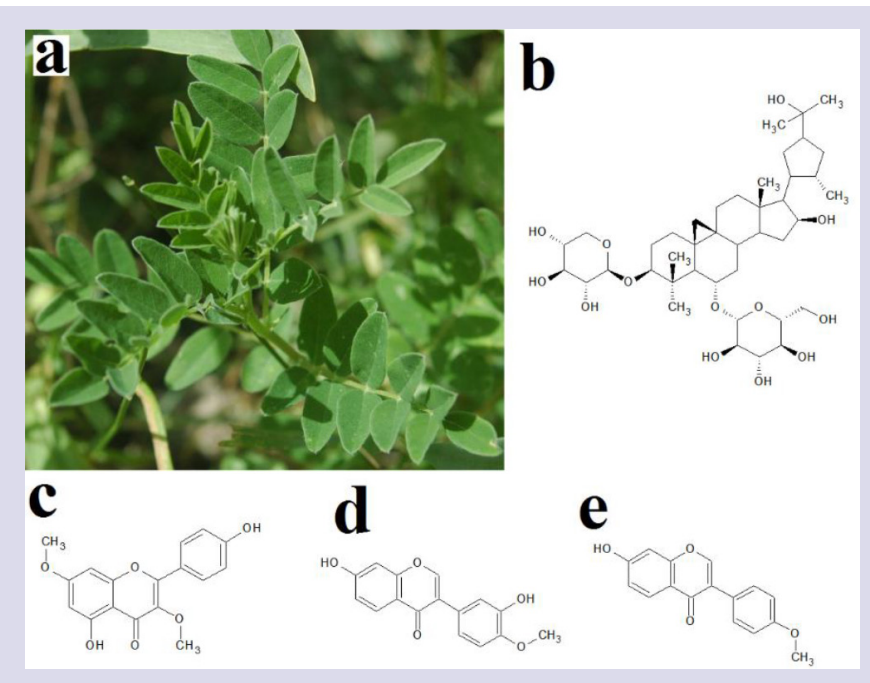

Figure 1: (a) A. membranaceus leaves and the structures of its common constituents: (b) the triperpenoid astragaloside and the isoflavones (c) kumatakenin, (d) calycosin, (e) formononetin. The photograph is open source and is reproduced from https://commons.wikimedia.org/wiki/ File:Astragalus_membranaceus.jpg with all relevant permissions.

normal components of the gut microbiome were therefore also included in the current study.

Adherents of complementary and alternative medicine often use both traditional and allopathic medications concurrently without any regard to the interactions between the medicines and the possible side effects which may occur. Potential combination interactions may pose serious risks to patient safety and a greater understanding of these effects is required. ${ }^{21-23}$ Notably, it has been estimated that up to approximately $70 \%$ of patients in Western countries use herbal drugs concurrently with prescription drugs. ${ }^{24-25}$ Furthermore, it is likely that the use of herbal therapies in combination with over-the-counter medications is substantially higher, with many patients combining these two medicinal systems believing that the combination would provide an enhanced effect. Often this self-prescribing occurs without the knowledge of their physician and there have been many instances where severe reactions have resulted. ${ }^{26}$ Much more work is required to test the effects of allopathic and complementary drug combinations on both the safety and efficacy of the combinations.

In this report, we tested Astragalus root powder extracted with solvents of varying polarity against diarhoea-causing Alcaligenes faecalis, Bacillus cereus, Salmonella newport, Aeromonas hydrophila, Escherichia coli and Shigella sonnei, all of which are known bacterial triggers of GI diseases. A. faecalis may cause systemic infections and symptomatic peritonitis which can be fatal if untreated. ${ }^{27}$ S. newport causes an estimated 1.2 million illnesses, 23,000 hospitalizations and 450 deaths annually and Salmonella is a serious threat to public health. ${ }^{28} B$. cereus produces mucin in the gut which induces the onset of pathogenesis. ${ }^{29} \mathrm{~S}$. sonnei is a major public health concern worldwide, especially in developing countries, with acute intestinal infections requiring a minimum infective dose as low as 10-100 bacterial cells, ${ }^{30}$ while $A$. hydrophila can induce intestinal mucosal barrier function damage and inflammation. ${ }^{31}$ The genus Escherichia consists of facultative anaerobic Gram-negative bacilli that belong to the family Enterobacteriaceae and although most E. coli strains live harmlessly in the colon and seldom cause disease in healthy individuals, a number of pathogenic strains can cause intestinal and extra-intestinal diseases, both in healthy and immunocompromised individuals. ${ }^{32}$ In contrast, Enterococcus faecalis and Enterobacter cloacae are harmless bacterial residents of the gastrointestinal microbiome. They have been included in this study to determine whether Astragalus is capable of inhibiting these non-pathogenic strains, which could ultimately alter the gut microbiome. The efficacy of Astragalus extracts, alone and in combination with conventional antibiotics, against five pathogens that trigger diarrhea and against two non-pathogenic GI bacteria, were assessed.

\section{MATERIALS AND METHODS}

\section{Collection of plant samples}

The Astragalus root powder used in this study was sourced from verified plants in China by Noodles Emporium (Australia) and supplied as a dried, ground powder. A voucher sample (NSC2017wsic) has been stored at the School of Environment and Science, Griffith University, Australia.

\section{Preparation of extracts}

Individual $1.5 \mathrm{~g}$ quantities of the material were weighed into separate tubes and deionised water, methanol, ethyl acetate, hexane or chloroform were added to $50 \mathrm{~mL}$. All organic solvents were obtained from Ajax, Australia and were AR grade. The ground plant materials were individually extracted in each solvent for $24 \mathrm{~h}$ at $41^{\circ} \mathrm{C}$ with gentle shaking. The extracts were subsequently filtered through filter paper (Whatman No. 54) under vacuum and dried at $42^{\circ} \mathrm{C}$, with the resultant extracts weighed and resuspended in $10 \mathrm{~mL}$ deionised water (containing $1 \% \mathrm{DMSO}$ ). The suspensions were briefly sonicated ( $3 \times 20 \mathrm{~s}$ pulse cycles, at $2 \mathrm{kHz}$ ), sterilised by filtration through a $0.2 \mu \mathrm{m}$ membranes and stored at $4{ }^{\circ} \mathrm{C}$ until required for further analysis.

\section{Qualitative phytochemical studies}

Phytochemical analyses of the Astragalus root extracts for the presence of saponins, phenolic compounds, flavonoids, phytosterols, triterpenoids, cardiac glycosides, anthraquinones, tannins and alkaloids were conducted by previously described assays. ${ }^{33}$

\section{Antibiotics}

Penicillin-G (potency of 1440-1680 $\mu \mathrm{g} / \mathrm{mg}$ ), chloramphenicol ( $\geq 98 \%$ purity by HPLC), erythromycin (potency $\geq 850 \mu \mathrm{g} / \mathrm{mg}$ ), ciprofloxacin ( $\geq 98 \%$ purity by HPLC) and tetracycline ( $\geq 95 \%$ purity by HPLC) were purchased from Sigma-Aldrich (Australia) and were used as controls for the microplate liquid dilution assay. All antibiotics were prepared in sterile deionised water at stock concentrations of $0.01 \mathrm{mg} / \mathrm{mL}$ and stored at $4^{\circ} \mathrm{C}$ until use.

\section{Bacterial cultures}

Reference strains of A. faecalis, B. cereus, A. hydrophila, S. sonnei, E. faecalis, E. coli and E. cloacae were purchased from the American Type Culture Collection (USA). The S. newport clinical isolate strain was obtained from the School of Environment and Science teaching laboratory. All bacteria were cultured in nutrient broth (Oxoid Ltd., Australia). Streaked nutrient agar (Oxoid Ltd., Australia) plates were tested in parallel to ensure the purity of all bacterial cultures and for sub-culturing.

\section{Disc diffusion assays on agar}

Antibacterial activity screening of the Astragalus root extracts on solid agar was assessed using a modified disc diffusion assay method. ${ }^{34-36}$ Briefly, single colonies of the test bacteria isolated from streaked agar plates were grown at $37^{\circ} \mathrm{C}$ in $40 \mathrm{~mL}$ of fresh nutrient broth media until they reached a count of approximately $10^{8}$ cells $/ \mathrm{mL}$. The visual turbidity of each culture was adjusted in order to prepare $0.5 \mathrm{McF}$ arland standards. A volume of $100 \mu \mathrm{L}$ of the individual microbial suspensions were spread onto nutrient agar plates. Extracts $(10 \mu \mathrm{L})$ were infused on 
Whatman \#1 filter discs (6 $\mathrm{mm}$ in diameter), with negative control discs containing $10 \mu \mathrm{L}$ of extract resuspension solvent ( $1 \% \mathrm{DMSO})$. Penicillin, erythromycin, ciprofloxacin, tetracycline and chloramphenicol discs $(1 \mu \mathrm{g})$ were prepared and used as positive controls to compare antibacterial activity, whilst filter discs infused with $10 \mu \mathrm{L}$ of distilled water were used as a negative control for the antibiotics. Discs were allowed to dry and were then applied to inoculated agar. The plates were incubated at $37^{\circ} \mathrm{C}$ for 18-24 h. The zone of inhibition (ZOI) was measured for each disc and was inclusive of the $6 \mathrm{~mm}$ diameter of the filter disc.

\section{Minimum inhibitory concentration (MIC) determination}

The minimum inhibitory concentration for each extract was determined using a microplate liquid dilution MIC method ${ }^{37-38}$ which is generally considered a highly sensitive bacterial growth inhibitory assay. Furthermore, as microplate liquid dilution MIC assays are perhaps the most commonly used method of quantifying bacterial growth inhibition efficacy, use of this method allows for comparisons with other studies. Briefly, $100 \mu \mathrm{L}$ of resuspended extract stocks or antibiotics $(1 \mu \mathrm{g})$ were added to $100 \mu \mathrm{L}$ of nutrient broth in the top row of 96-well microplates. Positive control lanes containing nutrient broth only, 1\% DMSO (control for extracts) or $\mathrm{dH}_{2} \mathrm{O}$ (control for antibiotics) were also prepared. Three-fold serial dilutions were prepared in each subsequent row, each containing $100 \mu \mathrm{L}$ of nutrient broth. Finally, $100 \mu \mathrm{L}$ of a $1: 100$ dilution of a 0.5 McFarland bacterial standard were added to each well and microplates were incubated at $37^{\circ} \mathrm{C}$ for $24 \mathrm{~h}$. p-Iodonitrotetrazolium violet (SigmaAldrich, Australia) dissolved in sterile deionised water to produce a 0.2 $\mathrm{mg} / \mathrm{mL}$ INT solution was prepared and a $40 \mu \mathrm{L}$ volume of this solution was added into all wells. Microplates were incubated for a further $6 \mathrm{~h}$ at $24-30^{\circ} \mathrm{C}$. Following incubation, the MIC was visually determined as the lowest dose at which colour development was inhibited.

\section{Fractional inhibitory concentration (FIC) assessment}

Interactions between the Astragalus root extracts and the conventional antibiotics were examined by determination of the sum of fractional inhibitory concentrations ( $\Sigma$ FIC) for each combination. ${ }^{39}$ The FIC values for each component ( $\mathrm{a}$ and $\mathrm{b}$ ) were calculated using the following equations where a represents the plant extract sample and $b$ represents the conventional antibiotic:

$$
\begin{aligned}
& \operatorname{FIC}(\mathrm{a})=\left(\frac{\text { MIC }[\mathrm{a} \text { in combination with } \mathrm{b}]}{\mathrm{MIC}[\mathrm{a} \text { independently }]}\right) \\
& \mathrm{FIC}(\mathrm{b})=\left(\frac{\mathrm{MIC}[\mathrm{b} \text { in combination with } \mathrm{a}]}{\mathrm{MIC}[\mathrm{b} \text { independently }]}\right)
\end{aligned}
$$

The $\Sigma F I C$ was then calculated using the formula $\Sigma F I C=F I C(a)+$ $\mathrm{FIC}(\mathrm{b})$. The interactions were classified as synergistic $(\Sigma \mathrm{FIC} \leq 0.5)$, additive $(\Sigma$ FIC $>0.5-1.0)$, indifferent $(\Sigma F I C>1.0-4.0)$ or antagonistic $(\Sigma \mathrm{FIC}>4.0){ }^{37-38}$

\section{Artemia nauplii toxicity screening}

An aqueous preparation $(4 \mathrm{mg} / \mathrm{mL})$ of potassium dichromate $\left(\mathrm{K}_{2} \mathrm{Cr}_{2} \mathrm{O}_{7}\right)$ (AR grade, Chem-Supply, Australia) was serially diluted in artificial seawater for use as a reference toxin. Toxicities of the Astragalus root extracts, the reference toxin and the conventional antibiotics were assessed using a modified Artemia franciscana nauplii lethality assay. ${ }^{40-41}$ The $\mathrm{LC}_{50}$ with $95 \%$ confidence limits for each treatment was calculated using probit analysis.

\section{Statistical analysis}

Data are expressed as the mean \pm SEM of at least three independent experiments, each with internal triplicates $(n=9)$. One way ANOVA was used to calculate statistical significance between control and treated groups with a $P$ value $<0.01$ considered as statistically significant.

\section{RESULTS}

\section{Liquid extraction yields and phytochemical screening}

Astragalus root extractions (1.5 g material) using various solvents yielded dried plant extracts ranged from $54 \mathrm{mg}$ to $245 \mathrm{mg}$ (Table 1). Aqueous,

Table 1: The mass of dried extracted material, concentration after resuspension in deionised water and qualitative phytochemical screenings for the Astragalus

\begin{tabular}{|c|c|c|c|c|c|c|c|c|c|c|c|c|c|c|c|c|c|c|}
\hline \multirow{2}{*}{ 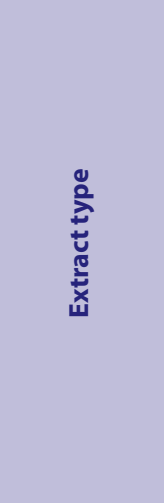 } & \multirow{2}{*}{ 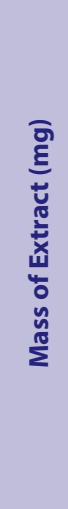 } & \multirow{2}{*}{ 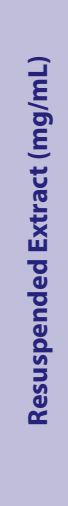 } & \multicolumn{3}{|c|}{$\begin{array}{l}\frac{u}{\bar{y}} \\
\frac{c}{0} \\
\frac{c}{\alpha}\end{array}$} & \multicolumn{2}{|c|}{ 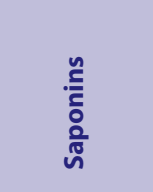 } & \multirow{2}{*}{ 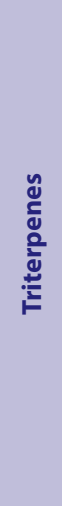 } & \multirow{2}{*}{ 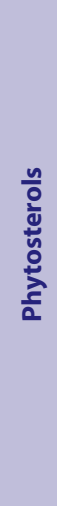 } & \multicolumn{3}{|c|}{ 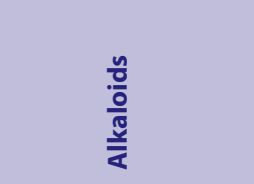 } & \multicolumn{2}{|c|}{$\begin{array}{l}\text { 끙 } \\
\frac{0}{0} \\
\frac{1}{0} \\
\frac{\pi}{0} \\
\frac{\pi}{4}\end{array}$} & \multicolumn{2}{|c|}{ 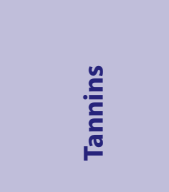 } & \multicolumn{2}{|c|}{ 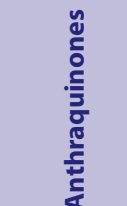 } \\
\hline & & & $\begin{array}{l}\text { సँ } \\
\text { 。ँ }\end{array}$ & $\begin{array}{l}\frac{0}{0} \\
\frac{0}{5} \\
\frac{0}{0} \\
\frac{1}{0} \\
\frac{1}{\pi} \\
3\end{array}$ & 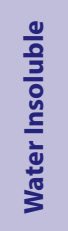 & 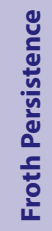 & 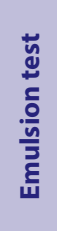 & & & 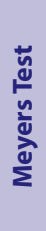 & $\begin{array}{l}\breve{y} \\
\frac{5}{n} \\
\frac{n}{2} \\
\frac{5}{\sigma} \\
\vdots\end{array}$ & 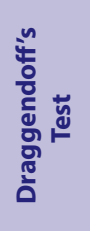 & 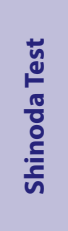 & 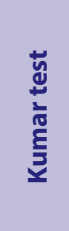 & 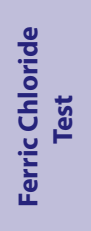 & 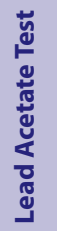 & $\begin{array}{l}\text { ॥ँ } \\
\text { Lั }\end{array}$ & 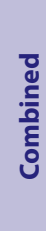 \\
\hline Methanol & 218 & 21.8 & +++ & +++ & +++ & + & + & - & - & - & - & - & +++ & +++ & ++ & ++ & - & - \\
\hline Water & 245 & 24.5 & +++ & +++ & +++ & ++ & ++ & - & - & - & - & - & +++ & +++ & ++ & ++ & - & - \\
\hline Ethyl Acetate & 54 & 5.4 & + & + & + & + & - & - & - & - & - & - & ++ & ++ & + & + & - & - \\
\hline Chloroform & 187 & 18.7 & + & + & + & - & - & - & - & - & - & - & + & - & - & - & - & - \\
\hline Hexane & 77 & 7.7 & - & - & - & - & - & - & - & - & - & - & - & - & - & - & - & - \\
\hline
\end{tabular}
root extracts.

+++ indicates a large response; ++ indicates a moderate response; + indicates a minor response; - indicates no response in the assay. 
methanolic and chloroform extracts provided significantly greater yields of extracted material relative to the hexane and ethyl acetate, which gave low to moderate yields. The dried extracts were resuspended in $10 \mathrm{~mL}$ of deionised water (containing $1 \%$ DMSO), resulting in the concentrations presented in Table 1. Qualitative phytochemical studies (Table 1) showed that methanol and water extracted the widest range and greatest relative abundance of phytochemicals. Both showed high levels of phenolics (both water soluble and insoluble phenolics) and flavonoids, as well as high to moderate to high levels of tannins and saponins. The ethyl acetate and chloroform extract also had low levels of phenolics and flavonoids. Moderate flavonoid levels were also detected in the ethyl acetate extract. No phytochemical classes were detected in the hexane extract.

\section{Quantification of minimum inhibitory concentration (MIC)}

Interestingly, none of the Astragalus extracts inhibited bacterial growth of any of the strains in the disc diffusion studies, indicating that the extracts are ineffective in the solid agar test model. However, zones of inhibitions were observed for the positive control antibiotics with varying efficacies, demonstrating that the assay was functioning correctly (Table 2). The antimicrobial efficacies of the Astragalus extracts were further assessed by determining the MIC values using liquid dilution assays. Only the aqueous extract showed inhibitory properties, with MIC values of $8167 \mu \mathrm{g} / \mathrm{ml}$ against $A$. faecalis, A. hydrophila, B. cereus, S. newport and $S$. sonnei (Table 3 ). Whilst inhibition was noted, these MIC values indicate only low antibacterial activity. None of the extracts showed activity towards E. cloacae, E. coli, or E. faecalis. MIC values for the positive control antibiotics against all strains in liquid dilution assays are shown in Table 3.

\section{Determination of combinational effects: Fractional inhibitory concentration (FIC) assessment}

Since only the aqueous extracts was found to possess activity against some bacterial strains, this extract was tested further against those strains in combination with the conventional antibiotics used in this study in order to determine the fractional inhibitor concentrations (FICs) of each combination. Thus, the methanol, ethyl acetate, hexane and chloroform Astragalus extracts and E. cloacae, E. coli and E. faecalis were excluded from this analysis as no activity was initially observed with either the extracts or these bacteria and therefore determination of $\Sigma$ FIC values is not possible. The $\Sigma$ FIC calculations were determined using a 1:1 ratio of the aqueous Astragalus extract with and without conventional antibiotics against the bacterial strains which were inhibited by the aqueous extract. The sums of FIC ( $\Sigma$ FIC) were calculated where possible and the class of interaction was determined for each. These values are shown in Table 4 . Additive interactions between the aqueous extract and penicillin, erythromycin, tetracycline and ciprofloxacin were observed against A. faecalis, with an additive interaction also observed between the aqueous extract and tetracycline or chloramphenicol for A. hydrophila. An additive interaction between the aqueous extract and chloramphenicol was also noted for B. cereus. These are promising findings since the antibacterial activity of these combinations is enhanced against these strains, albeit not to the extent expected for a synergistic interaction. However, the additive growth inhibitory effects of such combinations show that it would be preferable as a growth inhibitor of these bacteria compared to treatment with the individual antibiotic or extract components. Of the remaining combinations, seven were determined to be non-interactive. Whilst co-administering these extracts and antibiotics would be of no therapeutic benefit, they would not counteract/lessen the activity of the individual components and therefore may be deemed safe to use concomitantly.
Table 2: Zones of inhibition for the positive control antibiotics ( $1 \mu \mathrm{g}$ per disc) used in this study, as determined using agar disc diffusion assays. Zones include the diameter of disc $(6 \mathrm{~mm})$.

\begin{tabular}{|c|c|c|c|c|c|c|c|c|}
\hline & \multicolumn{8}{|c|}{ Zone of inhibition ( $\mathrm{mm}$ ) } \\
\hline & 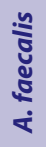 & <ن & ڤั & 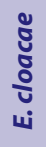 & 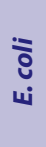 & 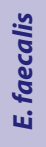 & 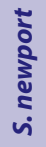 & 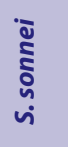 \\
\hline Pen & 7 & - & - & - & - & 9 & 9 & - \\
\hline Ery & 8 & - & - & - & - & 14 & - & - \\
\hline Tet & 9 & 11 & 8 & 12 & 11 & 14 & 7 & 12 \\
\hline Chl & - & 14 & - & - & 9 & 8 & - & 11 \\
\hline Cip & 18 & 28 & 16 & 29 & 28 & 15 & 18 & 26 \\
\hline
\end{tabular}

Pen $=$ penicillin; Ery = erythromycin; Tet $=$ tetracycline; $\mathrm{Chl}=$ chloramphenicol; Cip = ciprofloxacin; (-) indicates that no inhibition was detected.

Table 3: MIC values for aqueous Astragalus extract and the positive control antibiotics used in this study, as determined using liquid dilution assays.

\begin{tabular}{|c|c|c|c|c|c|c|c|c|}
\hline & & & Ant & ibacterial & I MIC ( $\mu \mathrm{g}$ & $/ / \mathrm{mL})$ & & \\
\hline & 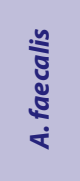 & $\begin{array}{l}\frac{0}{5} \\
\frac{0}{0} \\
\frac{0}{0} \\
\frac{0}{2}\end{array}$ & 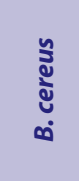 & 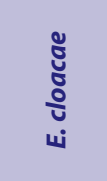 & نَ & 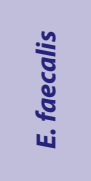 & 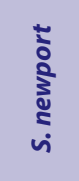 & 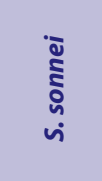 \\
\hline EXT & 8167 & 8167 & 8167 & - & - & - & 8167 & 8167 \\
\hline Pen & 0.11 & - & - & - & - & 0.11 & 0.037 & - \\
\hline Ery & 0.33 & - & - & - & - & 0.037 & 0.11 & - \\
\hline Tet & 0.11 & 0.012 & 0.11 & 0.11 & 0.037 & 0.012 & 0.03 & 0.012 \\
\hline Chl & - & 0.037 & - & - & 0.33 & - & - & 0.11 \\
\hline Cip & 0.012 & 0.00015 & 0.037 & 0.00015 & 0.00015 & 0.037 & 0.012 & 0.00015 \\
\hline
\end{tabular}

$\mathrm{EXT}=$ aqueous Astragalus extract; Ery = erythromycin; Tet = tetracycline; $\mathrm{Chl}=$ chloramphenicol; $\mathrm{Cip}=$ ciprofloxacin; values represented by a dash (-) indicate that an MIC could not be measured as there was no inhibition at the highest concentration tested.

Table 4: $\Sigma$ FIC values of Astragalus aqueous extracts in combination with conventional antibiotics against the bacterial strains in this study that were found to be inhibited by the extract.

\begin{tabular}{cccccc}
\hline Bacterial Strain & Pen & Ery & Tet & Chl & Cip \\
\hline A. faecalis & 1.0 & 1.0 & 1.0 & ND & 0.53 \\
A. hydrophila & ND & ND & 0.52 & 0.52 & 1.01 \\
B. cereus & ND & 9.12 & 1.59 & 1.0 & 1.44 \\
S. newport & CND & ND & CND & ND & CND \\
S. sonnei & ND & ND & 14.25 & 2.0 & BND \\
\hline
\end{tabular}

Pen = penicillin; Ery = erythromycin; Tet $=$ tetracycline; $\mathrm{Chl}=$ chloramphenicol; $\mathrm{Cip}=$ ciprofloxacin $. \mathrm{ND}=\Sigma \mathrm{FIC}$ could not be determined as the antibiotic was not inhibitory at any concentration tested; $\mathrm{CND}=\Sigma$ FIC could not be determined as the combination was not inhibitory, while the antibiotic alone was inhibitory; $\mathrm{BND}=\Sigma$ FIC could not be determined as either the combination, or the antibiotic alone, were inhibitory at all concentrations tested. Additive ( $>0.5$ to $\leq 1.0$ ); Indifferent $(>1.0$ to $\leq 4.0)$; Antagonistic $(>4.0)$. 


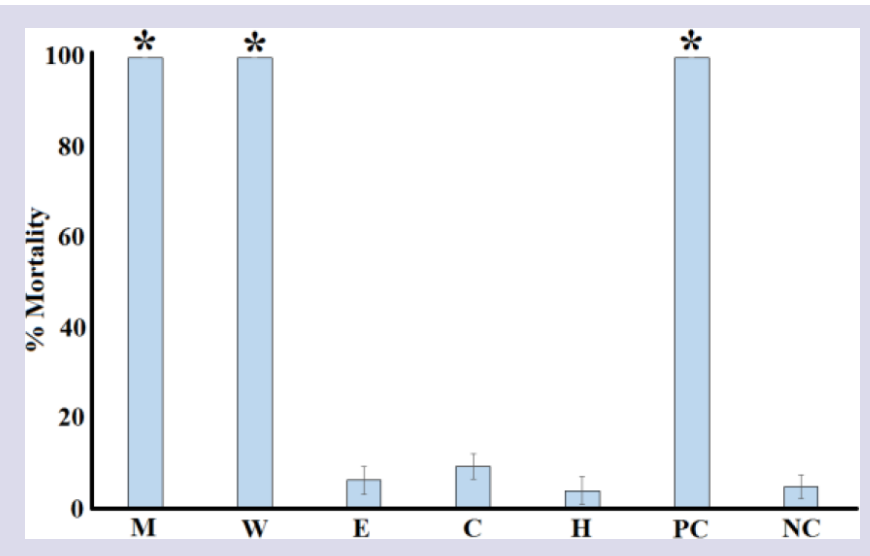

Figure 2: The lethality of the Astragalus extracts $(2000 \mu \mathrm{g} / \mathrm{mL})$ and the potassium dichromate $(1000 \mu \mathrm{g} / \mathrm{mL})$ and seawater controls towards $\mathrm{Ar}$ temia nauplii after 24 hours exposure. $\mathrm{M}=$ methanolic extract; $\mathrm{W}=$ aqueous extract; $\mathrm{E}=$ ethyl acetate extract; $\mathrm{C}=$ chloroform extract; $\mathrm{H}=$ hexane extract; $\mathrm{NC}=$ negative (seawater) control; $\mathrm{PC}=$ potassium dichromate control $(1000 \mu \mathrm{g} / \mathrm{mL})$. Results are expressed as mean $\%$ mortality \pm SEM. * indicates results that are significantly different to the untreated control $(p<0.01)$.

$\Sigma$ FIC values indicative of antagonism were found for the aqueous extract and erythromycin against $B$. cereus and between the aqueous extract and tetracycline against $S$. sonnei $(\Sigma \mathrm{FIC}>4.0)$. This suggests that these extract and antibiotic combinations significantly reduce the inhibitory effect of the conventional antibiotics on S. newport, B. cereus and S. sonnei, indicating that these specific Astragalus: antibiotic combinations should be avoided in order to maintain antibacterial activity. No synergistic interactions $(\Sigma \mathrm{FIC} \leq 0.5)$ were evident for any combination against any bacterial species.

\section{Quantification of toxicity}

All Astragalus extracts were tested in the Artemia nauplii assay at 2000 $\mu \mathrm{g} / \mathrm{mL}$ as an initial toxicity screen (Figure 2). In this assay, $\mathrm{LC}_{50}$ values $>1000 \mu \mathrm{g} / \mathrm{mL}$ are classified as non-toxic. ${ }^{40}$ Additionally, potassium dichromate was included in the bioassay as a reference toxin/positive control. The induction of mortality by potassium dichromate was rapid with substantial nauplii death evident within the first $3 \mathrm{~h}$ of exposure and $100 \%$ mortality by 5 hours of exposure (data not shown). The methanolic and aqueous extracts also induced $100 \%$ mortality following $24 \mathrm{~h}$ exposure, whilst all other extracts induced substantially $<50 \%$ mortality. The ethyl acetate, chloroform and hexane extracts were therefore deemed to be non-toxic. The methanolic and aqueous extracts were serially diluted in artificial seawater and further tested across a range of concentrations to determine their $\mathrm{LC}_{50}$ values. The $24 \mathrm{~h} \mathrm{LC}_{50}$ values of the methanolic and aqueous Astragalus root extracts towards A. franciscana nauplii were 1488 and $1165 \mu \mathrm{g} / \mathrm{mL}$ respectively. As these $\mathrm{LC}_{50}$ values are $>1000 \mu \mathrm{g} / \mathrm{mL}$, these extracts were also deemed to be non-toxic.

\section{DISCUSSION}

Astragalus has been used in TCM for a wide array of therapeutic applications. However, TCM preparations often contain a mixture of different plant materials ${ }^{42-43}$ and thus analysis of the individual components can prove difficult. Therefore, it is important to be able to study a single plant species. In the present study, we acquired a pure preparation of the Astragalus root powder which enabled us to analyse it in isolation.

Studies on the antibacterial properties of Astragalus are lacking. There- fore, one aim of this project was to investigate the efficacy of Astragalus (alone and in combination with conventional antibiotics) against pathogens which cause diarrhoea. Whilst we did detect some antibacterial activity in this study, most bacterial species examined were refractory to inhibition by the extracts. However, activity was observed for the aqueous Astragalus extract towards A. faecalis, A. hydrophila, B. cereus, S. newport and $S$. sonnei, which are known triggers of gastrointestinal diseases. This suggests that Astragalus contains components that could be used to treat illnesses that feature these disease-causing bacteria, despite the fact that the bacteriostatic/cidal effects are relatively mild. To our knowledge, this represents that first evidence that Astragalus possesses bacterial growth inhibitory properties, especially against these specific strains. Importantly, none of the plant extracts affected the growth of the normal gut flora strains tested in this study (E. cloacae, E. coli or E. faecalis). This indicates that Astragalus treatment may be used safely without inducing detrimental shifts in the gastrointestinal microbiome which may allow harmful/pathogenic bacteria to flourish.

The combinational studies reported in this manuscript screened the Astragalus extracts for interactions with the conventional antibiotics. These studies are not only important to identify possible antimicrobial alternatives to overcome bacterial antibiotic resistance; they may also provide valuable information for clinical use of these therapies, where herbal therapy-conventional antibiotic interactions may occur. Many people use herbal therapies and conventional antibiotics concurrently, without any understanding of the interactions that may occur between the different medicines. Natural products may have severe interactions in combination with conventional medicines, even when either component is safe when used alone. ${ }^{26}$ Also of concern, the efficacy of a therapy may be affected in combination with other medicines. Whilst, some combinations may have increased potency, other combinations may antagonize each other's effects, thereby decreasing the efficacy of the therapy. It is important to identify such combinations so that they are avoided.

Several additive combinatorial interactions were noted. In particular, the water extract potentiated the activity of chloramphenicol against $B$. cereus and of either tetracycline or chloramphenicol against $A$. hydrophila. That extract also potentiated the activity of penicillin, erythromycin, tetracycline and ciprofloxacin against A. faecalis. As such, these combinations would be beneficial against diarrhoea and dysentery, as they have greater efficacy than that of either component alone. Of the remaining combinations, the majority were either non-interactive, or were unable to be determined as at least one of the components in the combination showed no inhibition of bacterial growth when tested alone. Therefore, whilst using these combinations would have no additional therapeutic benefit, the components would not impede the activity of the other component. Notably, two combinations produced an antagonistic effect against $B$. cereus (extract with erythromycin) and $S$. sonnei (extract with tetracycline). These specific combinations should be avoided in the treatment of diarrhoea and any other diseases caused by these bacteria. The mechanisms of action regarding additive or antagonistic effects of the combinations against bacterial growth were not examined in this study. However, they may involve the alteration of specific cellular components by the plant extract to facilitate changes in the bacterial growth properties. Further investigations are necessary in order to more definitively identify the mechanism(s) involved.

Whilst a detailed investigation of the phytochemistry of the Astragalus extracts was beyond the scope of this study, the qualitative phytochemical studies highlighted several phytochemical classes that may contribute to the bacterial growth inhibitory activity and to the combinational effects noted in this study. The aqueous extracts had relatively high abundances in polyphenolics, saponins, flavonoids and tannins. The antibacterial activities are well known for a wide variety of flavonoids. ${ }^{44-45}$ Flavonoids 
inhibit bacterial growth via to a variety of mechanisms, including their ability to complex with extracellular and soluble proteins and as well as bacterial cell walls. ${ }^{46}$ Similarly, multiple tannins have broad antibiotic properties via a variety of mechanisms including binding, inactivating and/or precipitating of microbial proteins. ${ }^{47}$ Polyphenolic compounds are toxic to microorganisms via non-specific interaction with proteins or by reaction with sulfhydryl groups ${ }^{48}$ Therefore, phytochemical purification and structural analysis studies are required to evaluate the growth inhibitory mechanism(s) and to identify the bioactive and potentiating (or antagonizing) extract components.

\section{CONCLUSION}

The aqueous Astragalus extract possesses mild inhibitory effects on five different diarrhea and dysentery bacterial triggers. None of the plant extracts affect several harmless gut bacteria. Combinations of extracts with conventional antibiotics elicit several isolated cases of additive or antagonistic effects on bacterial growth inhibition and require further studies into the specific mechanisms responsible.

\section{ACKNOWLEDGEMENT}

Financial support for this work was provided by the Environmental Futures Research Institute and the Quality Use of Medicines (QUM) Network, Griffith University, Australia.

\section{CONFLICT OF INTEREST}

The authors declare no conflict of interest.

\section{ABBREVIATIONS}

DMSO: Dimethyl sulfoxide; $\mathbf{L C}_{50}$ : The concentration required to achieve 50 \% mortality; MIC: minimum inhibitory concentration.

\section{REFERENCES}

1. World Health Organization. Diarrhoeal disease. 2017. Retrieved from http://www.who.int/en/news-room/fact-sheets/detail/diarrhoeal-disease.

2. Amouzou A, Velez LC, Tarekegn H, Young M. One is too many: ending child deaths from pneumonia and diarrhoea. UNICEF. New York, NY. 2016;1-74.

3. Cheesman MJ, Ilanko A, Blonk B, Cock IE. Developing new antimicrobial therapies: Are synergistic combinations of plant extracts/compounds with conventional antibiotics the solution?. Pharmacognosy Reviews. 2017;11(22):57-72.

4. O'Neill J. Antimicrobial resistance: Tackling a crisis for the health and wealth of nations. Reviews on Antimicrobial Resistance. 2014;20:1-16.

5. Kapi A. The evolving threat of antimicrobial resistance: Options for action. Indian Journal of Medical Research. 2014;139(1):182-3.

6. Chan K. Chinese medicinal materials and their interface with Western medical concepts. Journal of Ethnopharmacology. 2005;96(1-2):1-18.

7. Liu P, Zhao H, Luo Y. Anti-aging implications of Astragalus membranaceus (Huangqi): a well-known Chinese tonic. Aging and Disease. 2017;8(6):868.

8. Zhang J, Xie X, Li C, Fu P. Systematic review of the renal protective effect of Astragalus membranaceus (root) on diabetic nephropathy in animal models. Journal of Ethnopharmacology. 2009;126(2):189-96.

9. Agyemang K, Han L, Liu E, Zhang Y, Wang T, Gao X. Recent advances in Astragalus membranaceus anti-diabetic research: Pharmacological effects of its phytochemical constituents. Evidence-Based Complementary and Alternative Medicine. 2013;2013:1-9.

10. Fu J, Wang Z, Huang L, Zheng S, Wang D, Chen S, Zhang H, Yang S. Review of the botanical characteristics, phytochemistry and pharmacology of Astragalus membranaceus (Huangqi). Phytotherapy Research. 2014;28(9):1275-83.

11. Bratkov VM, Shkondrov AM, Zdraveva PK, Krasteva IN. Flavonoids from the genus Astragalus: Phytochemistry and biological activity. Pharmacognosy Reviews. 2016;10(19):11-32.

12. Zhong $Y$, Deng $Y$, Chen $Y$, Chuang PY, He JC. Therapeutic use of traditional Chinese herbal medications for chronic kidney diseases. Kidney International. 2013;84(6):1108-18.

13. Jiang $X$, Cao $X$, Huang $Y$, Chen J, Yao X, Zhao M, et al. Effects of treatment with Astragalus membranaceus on function of rat leydig cells. BMC Complementary and Alternative Medicine. 2015;15(1):261.
14. Liao H, Hu L, Cheng $X$, Wang $X$, Li J, Banbury L, et al. Are the therapeutic effects of Huangqi (Astragalus membranaceus) on diabetic nephropathy correlated with its regulation of macrophage iNOS activity?. Journal of Immunology Research. 2017;2017:3780572.

15. Zhou R, Chen H, Chen J, Chen X, Wen Y, Xu L. Extract from Astragalus membranaceus inhibit breast cancer cells proliferation via PI3K/AKT/mTOR signaling pathway. BMC Complementary and Alternative Medicine. 2018;18(1):83.

16. Liu X, Yang Y, Zhang X, Xu S, He S, Huang W, et al. Compound Astragalus and Salvia miltiorrhiza extract inhibits cell invasion by modulating transforming growth factor- $\beta / S m a d$ in HepG 2 cell. Journal of Gastroenterology and Hepatology. 2010;25(2):420-6

17. Auyeung KKW, Mok NL, Wong CM, Cho CH, Ko JKS. Astragalus saponins modulate $\mathrm{mTOR}$ and ERK signaling to promote apoptosis through the extrinsic pathway in HT-29 colon cancer cells. International Journal of Molecular Medicine. 2010;26(3):341-9.

18. Li Z, Zhang L, He W. Zhu C, Yang J, Sheng M. Astragalus membranaceus inhibits peritoneal fibrosis via monocyte chemoattractant protein (MCP)-1 and the transforming growth factor- $\beta 1$ (TGF- $\beta 1$ ) pathway in rats submitted to peritoneal dialysis. International Journal of Molecular Sciences. 2014 15(7):12959-71.

19. Huang YC, Tsay HJ, Lu MK, Lin CH, Yeh CW, Liu HK, et al. Astragalus membranaceus-polysaccharides ameliorates obesity, hepatic steatosis, neuroinflammation and cognition impairment without affecting amyloid deposition in metabolically stressed APPswe/PS1dE9 mice. International Journal of Molecular Sciences. 2017;18(12):2746

20. Li J, Harata LY, Denton MD, Feng Q, Rathjen JR, Qu Z, et al. Long read reference genome-free reconstruction of a full-length transcriptome from Astragalus membranaceus reveals transcript variants involved in bioactive compound biosynthesis. Cell Discovery. 2017;3:17031.

21. Butterweck $V$, Derendorf $H$. Herb-drug interactions. Planta Medica 2012;78(13):1399.

22. Vieira MdLT, Huang SM. Botanical-drug interactions: a scientific perspective. Planta Medica. 2012;78(13):1400-15.

23. Lai W, Chen J, Cock IE, Cheesman MJ. The interactive antimicrobial activity of Withania somnifera (L.) Dunal root extracts and conventional antibiotics against some bacterial triggers of autoimmune inflammatory diseases. Pharmacognosy Communications. 2018;8(2):86-92

24. Singh SR, Levine MA. Natural health product use in Canada: analysis of the National Population Health Survey. Canadian Journal of Clinical Pharmacology. 2006;13(2):e240-50.

25. Tindle HA, Davis RB, Phillips RS, Eisenberg DM. Trends in use of complementary and alternative medicine by US adults: 1997-2002. Alternative Therapies in Health and Medicine. 2005;11(1):42-9.

26. Cock I. The safe usage of herbal medicines: counter-indications, cross-reactivity and toxicity. Pharmacognosy Communications. 2015;5(1):2-50.

27. Kahveci A, Asicioglu E, Tigen E, Ari E, Arikan H, Odabasi Z, et al. Unusual causes of peritonitis in a peritoneal dialysis patient: Alcaligenes faecalis and Pantoea agglomerans. Annals of Clinical Microbiology and Antimicrobials. $2011 ; 10(1): 12$

28. Medalla F, Gu W, Mahon BE, Judd M, Folster J, Griffin PM, et al. Estimated incidence of antimicrobial drug-resistant nontyphoidal Salmonella infections, United States, 2004-2012. Emerging Infectious Diseases. 2017;23(1):29-37.

29. Tsilia V, Kerckhof FM, Rajkovic A, Heyndrickx M, Van DWT. Bacillus cereus $\mathrm{NVH}$ 0500/00 can adhere to mucin but cannot produce enterotoxins during gastrointestinal simulation. Applied and Environmental Microbiology. 2016;82(1):289-96.

30. Koh XP, Chiou CS, Ajam N, Watanabe H, Ahmad N, Thong KL. Characterization of Shigella sonnei in Malaysia, an increasingly prevalent etiologic agent of local shigellosis cases. BMC Infectious Diseases. 2012;12(1):122.

31. Kong W, Huang C, Tang Y, Zhang D, Wu Z, Chen X. Effect of Bacillus subtilis on Aeromonas hydrophila-induced intestinal mucosal barrier function damage and inflammation in grass carp (Ctenopharyngodon idella). Scientific Reports. 2017;7(1):1588.

32. Gomes TA, Elias WP, Scaletsky IC, Guth BE, Rodrigues JF, Piazza RM, et al. Diarrheagenic Escherichia coli. Brazilian Journal of Microbiology. 2016;47:3-30.

33. Cock IE, Kukkonen L. An examination of the medicinal potential of Scaevola spinescens: Toxicity, antibacterial and antiviral activities. Pharmacognosy Research. 2011;3(2):85-94

34. Arkhipov A, Sirdaarta J, Rayan P, McDonnell P, Cock I. An examination of the antibacterial, antifungal, anti-Giardial and anticancer properties of Kigelia africana fruit extracts. Pharmacognosy Communications. 2014;4(3):62-76.

35. Cock I. Antimicrobial activity of Acacia aulacocarpa and Acacia complanta methanolic extracts. Pharmacognosy Communications. 2012;2(1):66-71.

36. Cock I, Van VS. Anti-Proteus activity of some South African medicinal plants: their potential for the prevention of rheumatoid arthritis. Inflammopharmacology 2014;22(1):23-36

37. Hübsch Z, Van ZR, Cock I, Van VS. Interactive antimicrobial and toxicity profiles of conventional antimicrobials with Southern African medicinal plants. South African Journal of Botany. 2014;93:185-97. 
38. Ilanko A, White A, Cock I. The interactive antimicrobial activity of conventional antibiotics and Petalostigma spp. extracts against bacterial triggers of some autoimmune inflammatory diseases. BMC Complementary and Alternative Medicine. 2017. In press

39. Hutchings A Cock IE. An interactive antimicrobial activity of Embelica officinalis Gaertn. fruit extracts and conventional antibiotics against some bacterial triggers of autoimmune inflammatory diseases. Pharmacognosy Journal. 2018;10(4):654-62

40. Ruebhart DR, Wickramasinghe W, Cock IE. Protective efficacy of the antioxidants vitamin E and Trolox against Microcystis aeruginosa and microcystin-LR in Artemia franciscana nauplii. Journal of Toxicology and Environmental Health, Part A. 2009;72(24):1567-75

41. Vesoul J, Cock I. An examination of the medicinal potential of Pittosporum phylliraeoides: Toxicity, antibacterial and antifungal activities. Pharmacognosy Communications. 2011;1(2):8-17.

42. Efferth T, Kaina B. Toxicities by herbal medicines with emphasis to traditional Chinese medicine. Current Drug Metabolism. 2011;12(10):989-96.

43. Liu Y, Liu J, Wu KX, Guo XR, Tang ZH. A rapid method for sensitive profiling of bioactive triterpene and flavonoid from Astragalus mongholicus and Astragalus membranaceus by ultra-pressure liquid chromatography with tandem mass spectrometry. Journal of Chromatography B. 2018;1085:110-18.

44. Cock I. The phytochemistry and chemotherapeutic potential of Tasmannia lanceolata (Tasmanian pepper): A review. Pharmacognosy Communications. 2013;3(4):13-25

45. Narayana KR, Reddy MS, Chaluvadi M, Krishna D. Bioflavonoids classification pharmacological, biochemical effects and therapeutic potential. Indian Journa of Pharmacology. 2001;33(1):2-16.

46. Kaur GJ, Arora DS. Antibacterial and phytochemical screening of Anethum graveolens, Foeniculum vulgare and Trachyspermum ammi. BMC Complementary and Alternative Medicine. 2009;9(1):30.

47. Buzzini P, Arapitsas P, Goretti M, Branda E, Turchetti B, Pinelli P, et al. Antimicrobial and antiviral activity of hydrolysable tannins. Mini Reviews in Medicinal Chemistry. 2008:8(12):1179-87.

48. Cowan MM. Plant products as antimicrobial agents. Clinical Microbiology Reviews. 1999;12(4):564-82.

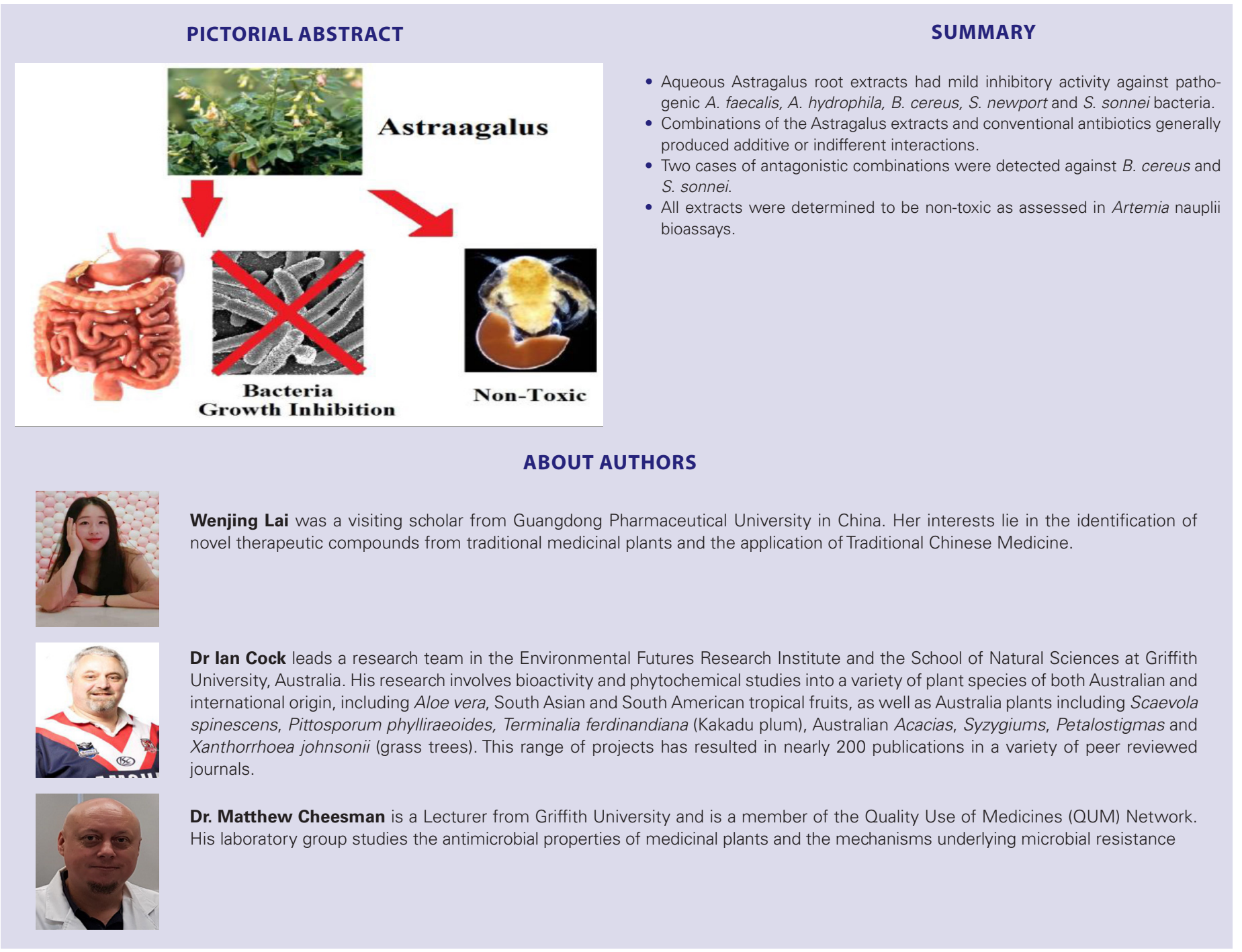

